\title{
Malignant transformation of hepatocellular adenoma with bone marrow metaplasia arising in glycogen storage disease type I: A case report
}

\author{
TOMOHIRO IGUCHI ${ }^{1}$, MOTOYUKI YAMAGATA ${ }^{2}$, TAKASHI SONODA ${ }^{2}$, KIMIHIKO YANAGITA ${ }^{3}$, \\ TETSUHIRO FUKAHORI ${ }^{4}$, EIJI TSUJITA ${ }^{1}$, SHINICHI AISHIMA ${ }^{5}$, YOSHINAO ODA ${ }^{6}$ and YOSHIHIKO MAEHARA $^{7}$ \\ ${ }^{1}$ Department of Gastroenterological Surgery, National Kyushu Cancer Center, Fukuoka 811-1395; Departments of ${ }^{2}$ Surgery, \\ ${ }^{3}$ Internal Medicine and ${ }^{4}$ Radiology, Saiseikai Karatsu Hospital, Karatsu, Saga 847-0852; ${ }^{5}$ Department of Pathology \\ and Microbiology, Saga Medical School Faculty of Medicine, Saga University, Saga 849-8501; Departments \\ of ${ }^{6}$ Anatomic Pathology and ${ }^{7}$ Surgery and Science, Graduate School of Medical Sciences, \\ Kyushu University, Fukuoka 812-8582, Japan
}

Received May 31, 2016; Accepted July 25, 2016

DOI: $10.3892 / \mathrm{mco} .2016 .1034$

\begin{abstract}
Malignant transformation of hepatocellular adenoma (HA) is relatively rare and has been reported to be associated with dysregulation of the $\beta$-catenin pathway. The presence of bone marrow metaplasia in HA is an uncommon histological characteristic. The current report presents the case of a 46-year-old woman with glycogen storage disease type I (von Gierke's disease) who underwent resection of hepatocellular carcinoma (HCC) arising in a HA with associated bone marrow metaplasia producing three series of hematopoietic cells. The serum level of proteins induced by des-gamma-carboxy prothrombin (DCP) gradually increased as the tumors grew; following hepatic resection, DCP levels returned to normal. Nuclear accumulation of $\beta$-catenin was shown in HCC by immunohistochemistry; however, no mutation was detected in exon 3 of $\beta$-catenin. To the best of our knowledge, this is the first report of HA with absolute bone marrow metaplasia producing three series of hematopoietic cells. This occurrence suggests that elevated DCP may be an indicator of malignant transformation of HA.
\end{abstract}

Correspondence to: Dr Tomohiro Iguchi, Department of Gastroenterological Surgery, National Kyushu Cancer Center, 3-1-1 Notame, Minami-ku, Fukuoka 811-1395, Japan

E-mail: tomo@surg2.med.kyushu-u.ac.jp

Abbreviations: HA, hepatocellular adenoma; DCP, des-gamma-carboxy prothrombin; GSD, glycogen storage disease; AFP, $\alpha$-fetoprotein; CT, computed tomography

Key words: hepatocellular carcinoma, hepatocellular adenoma, bone marrow metaplasia, des-gamma-carboxy prothrombin, $\beta$-catenin

\section{Introduction}

Hepatocellular adenoma (HA) is a benign tumor usually associated with oral contraceptive intake, glycogen storage disease (GSD) type I and III, and a history of excess androgen exposure (1-4). Malignant transformation of HA is relatively rare and has been reported to be associated with dysregulation of the $\beta$-catenin pathway $(2,5,6)$. Nuclear translocation and accumulation of $\beta$-catenin is induced by a dysregulation, such as a mutation of exon 3 (7), and may be detected by immunohistochemistry. The presence of bone marrow metaplasia in HA is an uncommon histological characteristic, with only 2 cases of HA with bone marrow metaplasia reported to date $(8,9)$.

We herein report a case with increased levels of proteins induced by des-gamma-carboxy prothrombin (DCP), resected hepatocellular carcinoma (HCC) and HA with bone marrow metaplasia arising in a patient with GSD-I.

\section{Case report}

History. A 46-year-old woman was diagnosed with GSD-I during childhood. From that time onwards, she was followed up in a local hospital and received treatment with atorvastatin and allopurinol. At the age of 42 years, the patient consulted a physician at the Saiseikai Karatsu Hospital (Karatsu, Japan), as she continued to experience developmental disorders, such as short stature $(135 \mathrm{~cm})$ and low weight $(36 \mathrm{~kg})$, but was asymptomatic, apart from abdominal enlargement. The liver edge was palpable $6-7 \mathrm{~cm}$ below the right costal margin. The patient had no other risk factors for liver tumors, such as hepatitis $\mathrm{B}$ or $\mathrm{C}$ viral infection, alcohol use, or autoimmune disease.

Liver function and tumor markers. Laboratory data at the first consult revealed elevated values of aspartate aminotransferase (96 IU/1), alanine aminotransferase (69 IU/1), alkaline phosphatase $(700 \mathrm{U} / 1)$, gamma-glutamyl transpeptidase (2610 IU/l) and DCP (421 mAU/ml). Serum total bilirubin, 
prothrombin time and $\alpha$-fetoprotein (AFP) were all within normal limits. Until surgical treatment was performed, while AFP remained within normal limits, DCP gradually increased, as shown in Fig. 1.

Imaging. A computed tomography (CT) examination at the first consult revealed relatively well-circumscribed tumors of various sizes (Fig. 2A and B) exhibiting heterogeneous enhancement during the early phase. During the delayed phase, some of the tumors in the lateral segment or the right hepatic lobe exhibited isodensity or low density compared with the surrounding liver parenchyma, whereas the small tumors in the medial segment exhibited low density. These imaging findings were compatible with the characteristics of HA. The size and number of tumors in the medial segment had significantly increased when compared to the CT from 33 months earlier (Fig. 2C and D). In addition, calcification was found in the tumor of S1.

Surgical treatment. Due to the gradual increase in tumor size and the value of DCP (maximum, 10,100 mAU/ml) and irregular tumor appearance on CT imaging, malignant transformation of HA was strongly suspected. According to the indication for hepatic resection, surgery was recommended, but the patient refused this treatment option for 1 year; when surgery was eventually performed, she underwent extended left lobectomy. Following hepatic resection, DCP returned to normal $(28 \mathrm{mAU} / \mathrm{ml})$.

Pathological findings. The resected left lobe weighed 2,733 g. The tumors were divided into four components. In brief, the cut surfaces revealed a dark green tumor $(4.7 \times 3.1 \mathrm{~cm})$ in $\mathrm{S} 1$, a dark green tumor $(10.0 \times 8.5 \mathrm{~cm})$ in the lateral segment, a gray colored tumor $(6.3 \times 5.0 \mathrm{~cm})$ in the lateral segment, and multiple pale yellow or white confluent nodules $(8.2 \times 7.2 \mathrm{~cm})$ in the medial segment, as shown in Fig. 3. Smaller pale yellow or white nodules were also observed. All the tumors were encapsulated by fibrous bands.

Microscopically, all the tumors, apart from the pale yellow or white masses, exhibited proliferation of small to occasionally large hepatocytes, with clear or pale eosinophilic cytoplasm and mild atypia, surrounded by numerous abnormal arterial vessels and no portal tract (Fig. 4A and B). These characteristics indicated HA. It was particularly worth noting that bone marrow tissue with bone trabeculae and three series of hematopoietic cells, namely erythroblasts, myeloblasts and megakaryocytes, was also present in the tumor of S1 (Fig. 4C and D).

The pale yellow or white tumors in the medial segment exhibited increased cell density and structural atypia. These tumor cells transformed into atypical cells with relatively enlarged hyperchromatic nuclei and increased nuclear-cytoplasmic ratio (Fig. 4E and F). Intrahepatic metastases were also present in the form of scattered small nodules consisting of atypical cells. These characteristics indicated well-to-moderately differentiated HCC, accompanied by HA. In addition, nuclear accumulation of $\beta$-catenin was observed in the carcinoma cells (Fig. 4G), but not in the adenoma cells (data not shown) by immunohistochemistry using anti- $\beta$-catenin mouse monoclonal antibody (dilution, 1:200; cat. no. 610154; BD Transduction Laboratories, Lexington, KY, USA). However, no mutation was detected in exon 3 of $\beta$-catenin by direct sequencing (data not shown).

The non-tumorous liver comprised hepatocytes with predominantly clear or pale cytoplasm, reflecting the accumulation of glycogen and glycogenated nuclei separated by portal-portal or portal-central thin bridging fibrosis (Fig. 4H). This characteristic was compatible with GSD-I.

\section{Discussion}

HA is a relatively rare benign liver tumor that mainly occurs in women who use oral contraceptives $(1,2)$. GSD-I is a rare metabolic disorder caused by deficient activity of glucose-6-phosphatase, an enzyme necessary for gluconeogenesis and glycogenolysis (10). GSD-I has been reported to be a cause of HA (3). An adenoma-carcinoma progression sequence may be expected in HCC, similar to colon cancer (11).

Bone marrow metaplasia in HA is an unusual characteristic, with only 2 cases reported to date $(8,9)$. Romacciato et al reported a case of HA where the bone marrow contained erythroblasts and myeloblasts, but not megakaryocytes. To the best of our knowledge, this is the first case report of an HA with absolute bone marrow metaplasia, producing three series of hematopoietic cells, incorporating a HCC. Hepatic progenitor cells, the preparative mature hepatocytes or cholangiocytes, have been identified in HA (12). As previously noted, marrow-derived hepatic stem cells may transform to both the atypical hepatocytes of HA and bone marrow differentiated cells (9).

The DCP and AFP values are well-known tumor markers of HCC. In the present case, AFP was within normal limits during disease progression; however, DCP was markedly elevated, consistently with increased tumor size, and it returned to normal after surgery. The development of HA or its malignant transformation in GSD-I patients is not necessarily associated with the value of serum $\operatorname{AFP}(13,14)$, whereas elevation of the serum DCP levels has been reported in a small number of patients with HA with malignant transformation (15). Therefore, the value of DCP may be useful for predicting the malignant transformation of HA.

Malignant transformation of HA is relatively rare and has been reported to be associated with dysregulation of $\beta$-catenin $(2,5,6)$, which plays an important role in the Wnt signaling pathway (7). However, a previous study revealed that neither nuclear accumulation of $\beta$-catenin detected by immunohistochemistry, nor mutation in exon 3 of $\beta$-catenin detected by direct sequencing, were observed in patients with HA, or HCC with HA (3). In our case, nuclear accumulation of $\beta$-catenin was observed on immunohistochemical examination, but no mutation in exon 3 of $\beta$-catenin was detected in HCC. The activation of the Wnt signaling pathway due to factors other than mutation of $\beta$-catenin may have occurred during malignant transformation in the present case. Therefore, malignant transformation of HA is unlikely to follow the same pathway.

We herein report malignant transformation in a patient with increased DCP levels, who underwent resection of HCC arising from HA with bone marrow metaplasia producing 


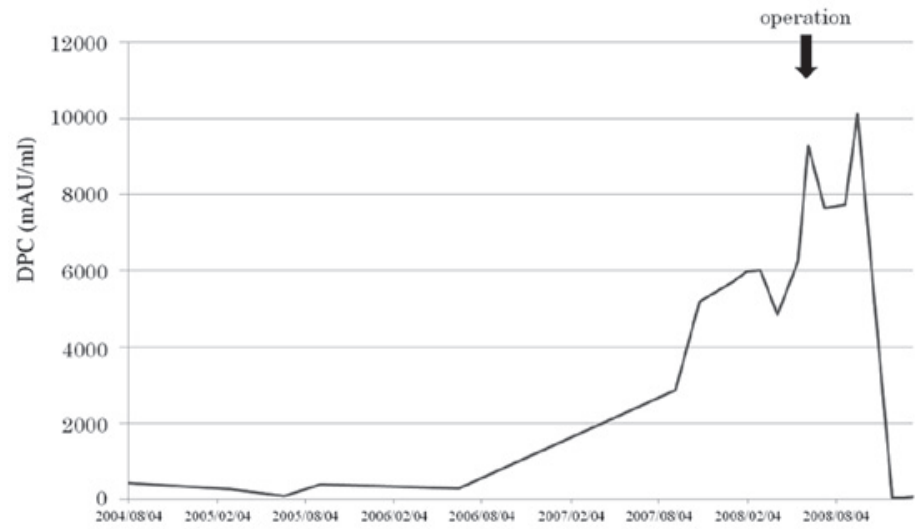

Figure 1. Transition of serum levels of des-gamma-carboxy prothrombin (DCP) from the initial consult to 1 month postoperatively. DCP increased gradually (maximum, 10,100 mAU/ml) until hepatic resection. Following hepatic resection, DCP decreased to $28 \mathrm{mAU} / \mathrm{ml}$, which is within normal limits.
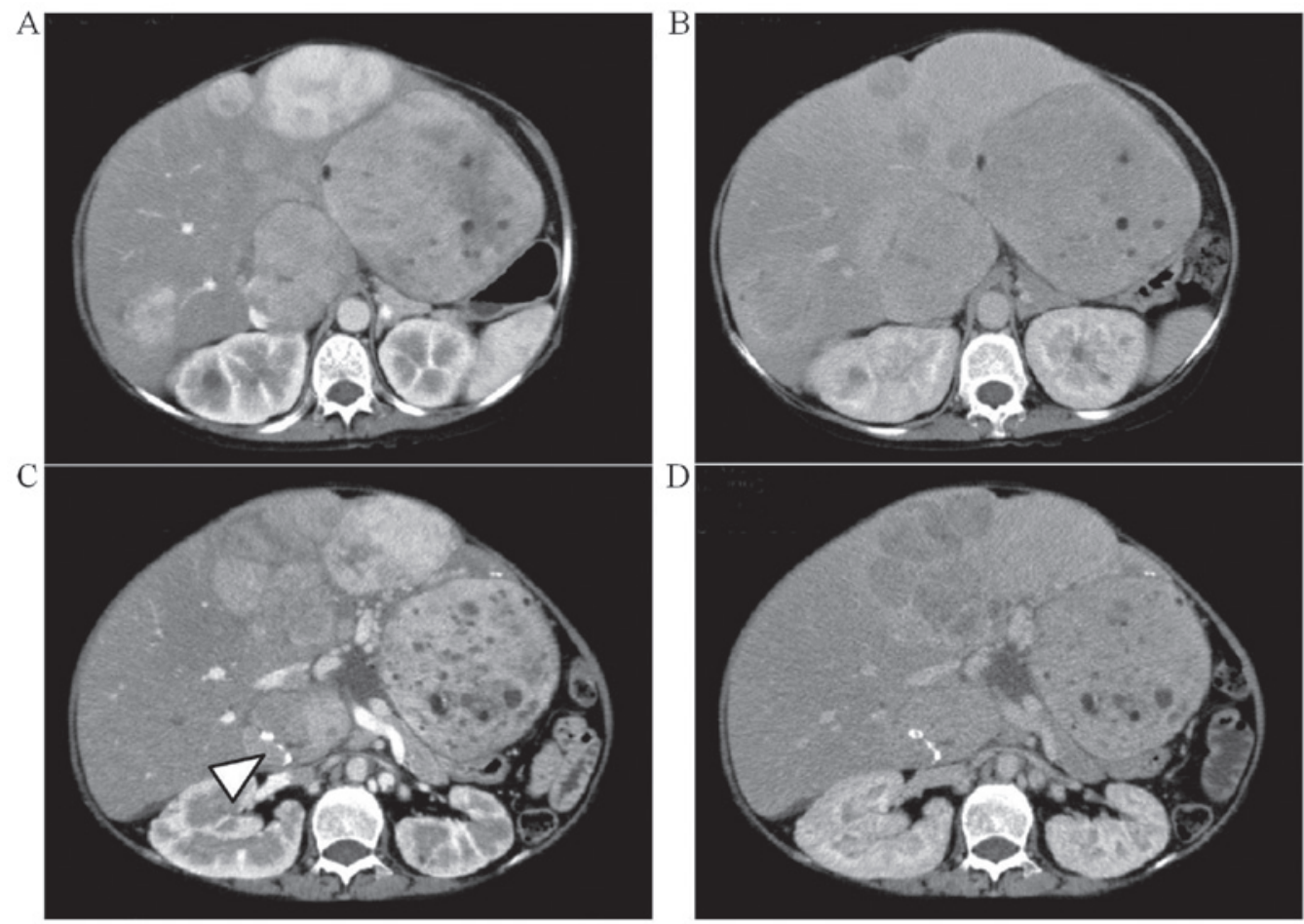

Figure 2. Computed tomography (CT) scans taken (A and B) at the first consult and (C and D) on the preoperative day. (A and C) Early-phase and $(\mathrm{B}$ and $\mathrm{D})$ delayed-phase tumors. The size and number of tumors in the medial segment had increased significantly and calcification was found in the tumor of S1 (arrowhead).

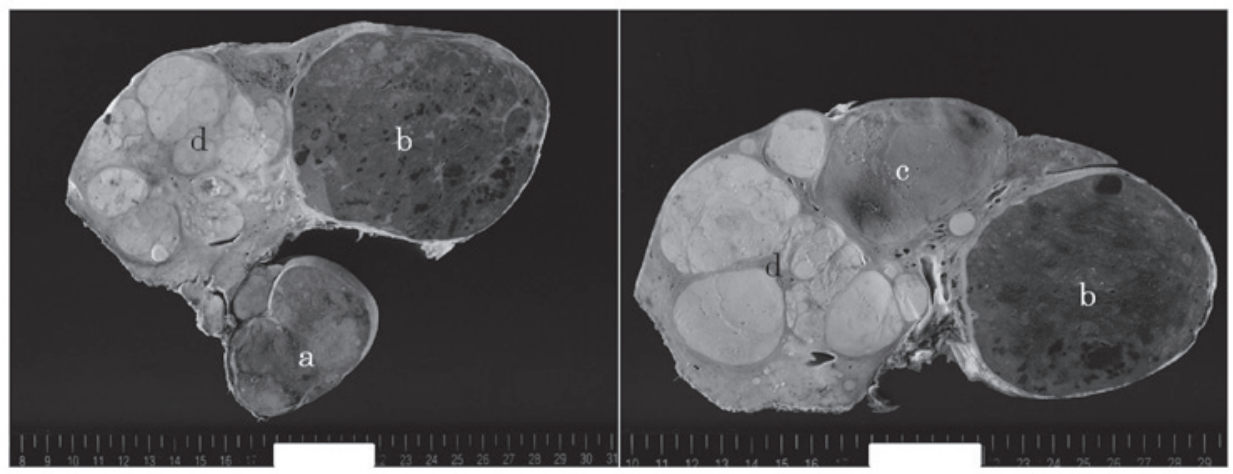

Figure 3. The representative cut surfaces revealed a dark green tumor $(4.7 \times 3.1 \mathrm{~cm})$ in S1 (a), a dark green tumor $(10.0 \times 8.5 \mathrm{~cm})$ in the lateral segment (b), a gray tumor $(6.3 \times 5.0 \mathrm{~cm})$ in the lateral segment $(\mathrm{c})$ and multiple pale yellow or white confluent nodules $(8.2 \times 7.2 \mathrm{~cm})$ in the medial segment (d). In addition, small pale yellow or white nodules were observed. 

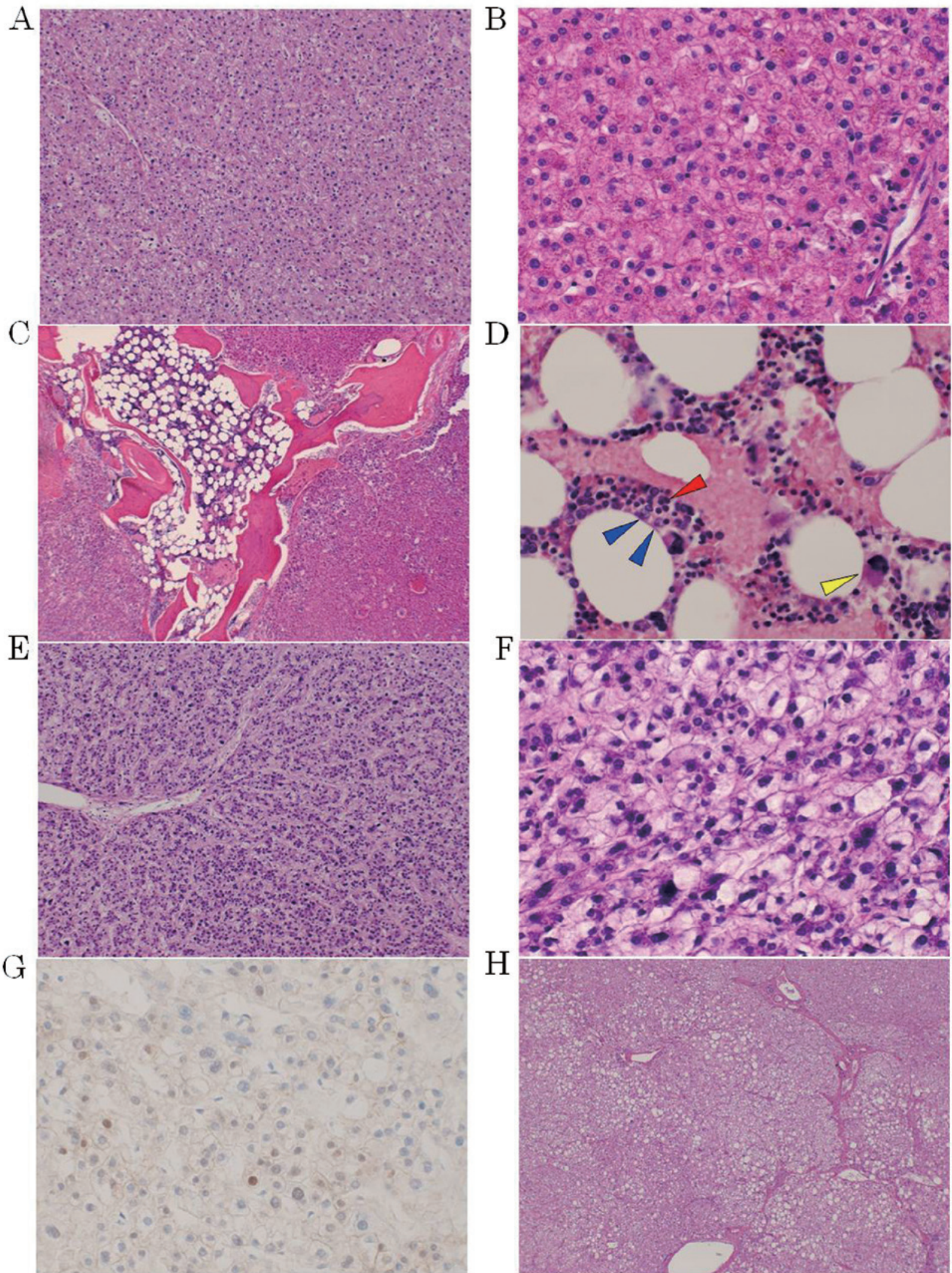

$\mathrm{H}$

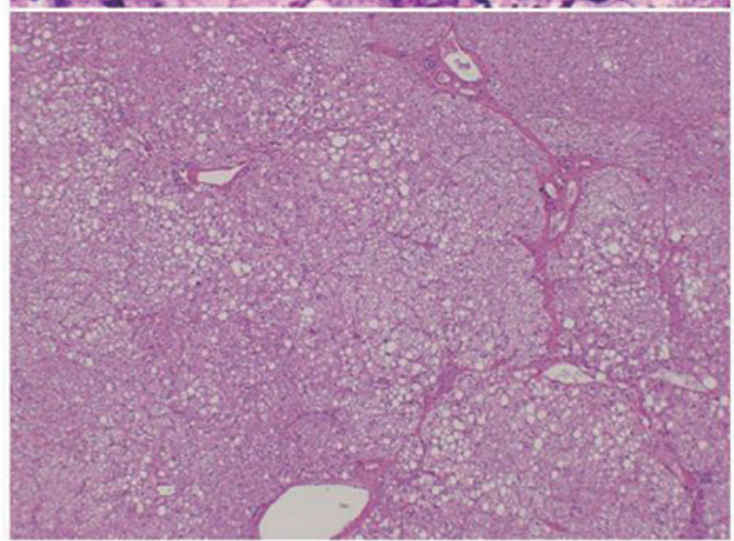

Figure 4. (A and B) Histological characteristics of the hepatocellular adenoma. (C and D) Bone marrow metaplasia: lamellar bone forming a trabecula, adipose tissue, erythroblasts (red arrowhead), myeloblasts (blue arrowhead) and megakaryocytes (yellow arrowhead). (E and F) Histological characteristics of hepatocellular carcinoma (HCC) arising in hepatocellular adenoma. (G) Immunohistochemical staining of $\beta$-catenin in HCC. (H) Non-tumorous liver. Original magnifications: (C) x20; (H) x40; (A and E) x100; (B, F and G) x200; and (D) x400.

three series of hematopoietic cells, based on GSD-I. The increased size and number of cells, as well as the elevated
DCP levels, may be considered as indicators of the malignant transformation of HA. 


\section{References}

1. Edmondson HA, Henderson B and Benton B: Liver-cell adenomas associated with use of oral contraceptives. N Engl J Med 294: 470-472, 1976.

2. Rebouissou S, Bioulac-Sage P and Zucman-Rossi J: Molecular pathogenesis of focal nodular hyperplasia and hepatocellular adenoma. J Hepatol 48: 163-170, 2008.

3. Micchelli ST, Vivekanandan P, Boitnott JK, Pawlik TM, Choti MA and Torbenson M: Malignant transformation of hepatic adenomas. Mod Pathol 21: 491-497, 2008.

4. Buell JF, Tranchart H, Cannon R and Dagher I: Management of benign hepatic tumors. Surg Clin North Am 90: 719-735, 2010

5. Agrawal S, Agarwal S, Arnason T, Saini S and Belghiti J: Management of hepatocellular adenoma: Recent advances. Clin Gastroenterol Hepatol 13: 1221-1230, 2015.

6. Zucman-Rossi J, Jeannot E, Nhieu JT, Scoazec JY, Guettier C, Rebouissou S, Bacq Y, Leteurtre E, Paradis V, Michalak S, et al: Genotype-phenotype correlation in hepatocellular adenoma: New classification and relationship with HCC. Hepatology 43: 515-524, 2006.

7. Polakis P: The oncogenic activation of beta-catenin. Curr Opin Genet Dev 9: 15-21, 1999

8. Moriura S, Kuroda M, Kimura A, Iwatsuka Y, Ikeda S, Sakai T and Usui A: Case report: Hepatic adenoma with bone marrow metaplasia in a patient with glycogen storage disease type Ia J Gastroenterol Hepatol 11: 556-559, 1996.
9. Ramacciato G, Nigri GR, Aurello P, D'Angelo F, Pezzoli F, Rossi S, Pilozzi E, Ercolani G and Ravaioli M: Giant hepatic adenoma with bone marrow metaplasia not associated with oral contraceptive intake. World J Surg Oncol 25: 4, 2006.

10. Ishak KG, Sharp HL and Schwarzenberg SJ: Metabolic errors and liver disease. In: MacSween RNM, Burt AD, Portmann BC, Ishak KG, Scheuer PJ, Anthony PP, editors. Pathology of the liver. London: Churchill Livingstone, 2002.

11. Gordon SC, Reddy KR, Livingstone AS, Jeffers LJ and Schiff ER: Resolution of a contraceptive-steroid-induced hepatic adenoma with subsequent evolution into hepatocellular carcionoma. Ann Intern Med 105: 547-549, 1986.

12. Libbrecht L, De Vos R, Cassiman D, Desmet V, Aerts R and Roskams T: Hepatic progenitor cells in hepatocellular adenomas. Am J Surg Pathol 25: 1388-1396, 2001.

13. Bianchi L: Glycogen storage disease I and hepatocellular tumours. Eur J Pediatr 152 (Suppl 1): S63-S70, 1993.

14. Lee PJ: Glycogen storage disease type I: Pathophysiology of liver adenomas. Eur J Pediatr 161 (Suppl 1): S46-S49, 2002.

15. Ito M, Sasaki M, Wen CY, Nakashima M, Ueki T, Ishibashi H, Yano M, Kage $\mathbf{M}$ and Kojiro M: Liver cell adenoma with malignant transformation: A case report. World J Gastroenterol 9: 2379-2381, 2003 